\title{
Simultaneous Loading and Injection Chip to Automate Single-cell Injections for Bovine Oocytes
}

\author{
Kevin Theodric Uning, Keita Ichikawa, Akiho Hirao, Taiga Michimoto, Tasuku Sato, \\ Hiroaki Kume, Takumi Nishida, Shota Iwakawa, and Yoko Yamanishi*
}

\author{
Department of Mechanical Engineering, Kyushu University, \\ 744 Motooka, Nishi-ku, Fukuoka-shi, Fukuoka-ken 819-0395, Japan
}

(Received July 14, 2020; accepted September 29, 2020)

Keywords: microinjection, bovine oocyte, rack and pinion micromagnetic tool, microbubble injector, in-chip injection

In order to simplify and automate the microinjection process, we propose a new microinjection chip for 100 - to $150-\mu \mathrm{m}$-size spherical cells, which integrates a novel rack-pinion loader and a previously developed bubble injector. Microinjection has always been the backbone of intracellular cargo delivery owing to its reliability for applications ranging from gene injection to intracellular drug delivery. Unfortunately, the conventional microinjection method is no longer sufficient as it requires highly skilled operators and is time-consuming. To solve these problems, we propose a novel reliable and reusable rack-pinion-based loader combined with a bubble injector for in-chip injection. Here, the rack-pinion loader was tested for its strength, precision, and loading capability, while the bubble injector was tested for its intercellular delivery capability. The rack-pinion-based loader has high performance under an external magnetic field, loading up to 42 particles per min. Furthermore, the pinion can be accurately positioned within $3^{\circ}$ and produces a $40 \mathrm{mN}$ force to deliver cells and overcome friction. The bubble injector entails a simple procedure with an 80 to $87.5 \%$ successful injection rate. Overall, the chip has a competitive throughput of at least 6 cells per min, including both the loading and injection processes.

\section{Introduction}

Microinjection has played major roles in intracellular delivery, supporting the rapidly advancing fields of biology, but its time-consuming procedure has been limiting the progress of these fields and thus motivating the development of alternatives. Since more than a century ago, when the microinjection technique was first introduced, it has supported numerous other fields, ranging from gene editing to human infertility treatment. ${ }^{(1)}$ Unfortunately, the technique itself cannot keep up with the growth and demand of biological research fields owing to its need for highly trained operators and its time-consuming and invasive nature. ${ }^{(2-5)}$ This situation has encouraged the development of various other methods of intracellular delivery,

*Corresponding author: e-mail: yoko@mech.kyushu-u.ac.jp

https://doi.org/10.18494/SAM.2020.2980 
such as electroporation and the use of photothermal nanoblades, viral vector-mediated systems, and bubble injectors. ${ }^{(6-9)}$ Among these methods, two of the most promising methods are electroporation and the use of bubble injectors, with electroporation being the better known owing to its generally high throughput and high efficiency.

The application of electroporation, however, is still limited to small cells as it requires a higher voltage to be applied to larger cells. Unlike small cells, large cells such as bovine and pig oocytes require more space between electrodes owing to their larger diameter. This means that in order to produce a similar electric field, a higher voltage must be applied between two electrodes, which greatly reduces the cell viability. ${ }^{(10)}$ Additionally, the voltage must be increased even further depending on the size of the cargo to be transported, ${ }^{(1)}$ further reducing the cell viability. As a result, the electroporation of large cells involves a significantly higher voltage, reducing the applicability of the current electroporation technology to the intracellular transportation of large cells.

On the other hand, the use of a bubble injector is more advantageous for large-cell injections, one reason being that it involves a procedure similar to microinjection. First, just like microinjection, the operation of a bubble injector is straightforward; it pierces the cell membrane with bubbles and injects the solution into the cell. ${ }^{(12)}$ Because of this simplicity, the operation of a bubble injector does not depend on the cell size or shape. Second, a bubble injector has a wide dynamic range of penetration capabilities as it does not physically touch the injection subject, ${ }^{(12)}$ expanding its range of application fields to include cell injection through plant cell walls. ${ }^{(13)}$ Third, a bubble injector is easy to automate because it can remain stationary for injections, simplifying the control of the injection. Finally, the use of a bubble injector also has a lower toxicity than other injection methods, increasing the efficiency of intracellular delivery. ${ }^{(14,15)}$ Therefore, a bubble injector is a compelling choice for the automation of single-cell injections, especially for large cells where electroporation cannot be easily used. In this paper, we propose a gene introduction chip with a rack-pinion loader and a bubble injector, in which successful injection was confirmed by the introduction of fluorescent beads into the cell. Moreover, we suggested that the proposed loading system can work well even under the harsh conditions of a cell culture medium.

\section{Concept Design}

In order to simplify and automate the operating procedure of bubble injectors, we propose a novel combination of a polydimethylsiloxane (PDMS) chip, a rack-pinion loader, and a bubble injector. Although microinjection has been simplified to a certain degree using the bubble injector, the injection procedure still faces a major problem in capturing cells effectively. To simplify this procedure, it is important to reduce the cell movement from three degrees of freedom (DOFs) to two or one DOF, which is commonly performed by using microchannels in PDMS chips. ${ }^{(16-18)}$ Unfortunately, the use of a PDMS chip by itself is still insufficient for intercellular transfer by bubble injection as the cells need to be delivered individually to the bubble injector. In order to solve this problem, we propose the use of our novel rackpinion loader inside the PDMS chip to deliver the cells individually to the injector. That is, by 
utilizing the gap between the teeth of the pinion, each cell can be separated in each gap and sent individually to the injector; this process is also known as loading. By combining these two components, the procedure for both positioning and injecting cells can be simplified into a series of one-DOF movements, resulting in an easy-to-operate system.

In addition to the simplified control, the rack-pinion loader design is also advantageous over other loaders, making it a compelling choice for this injection chip. First, compared with other gear-shaped microtools, ${ }^{(18-20)}$ the rack-pinion loader reacts more readily and strongly to external magnetic fields because of its large size. That is, the large size of this tool allows it to capture more magnetic field lines, which in turn causes a stronger interaction between the tool and the actuator. Second, the rack-pinion loader also has the capability to convert linear motion to rotational motion, resulting in a more efficient operation. Unlike a typical gear actuator, which relies on the complex circular motion of external magnets, the addition of the rack helps the operation become much simpler and smoother as it only involves one-DOF movements. Third, the large size of the pinion also allows multiple cells to be delivered within one cycle, allowing the pinion to rotate more slowly while keeping the cells safe and maintaining the throughput. As a result, the loading operation is simple, efficient, and safe for the cells, which makes the rack-pinion loader a suitable design for this application.

Combined with the rack-pinion loader, the bubble injector is also simple and easy to use. ${ }^{(21)}$ Its procedure can be described in two stages, as shown in Fig. 1. First, after a cell is locked in place in front of the injector, a high voltage is applied between the injector and the opposite electrode for a short duration. This generates bubbles in front of the injector and shoots the particles in front of the injector into the cell owing to the sudden increase in pressure. ${ }^{(22)}$ As a result, not only will this open a hole, it will also insert the nearby injection solution into the cell, completing the injection without any further action from the operator.

After setting up the two main components in the chip, the cells are injected by their insertion into the chip, followed by loading, then the injection process as shown in Fig. 2. First, the cells are inserted into the chip through the hole on the left. Then, these cells are transported to the

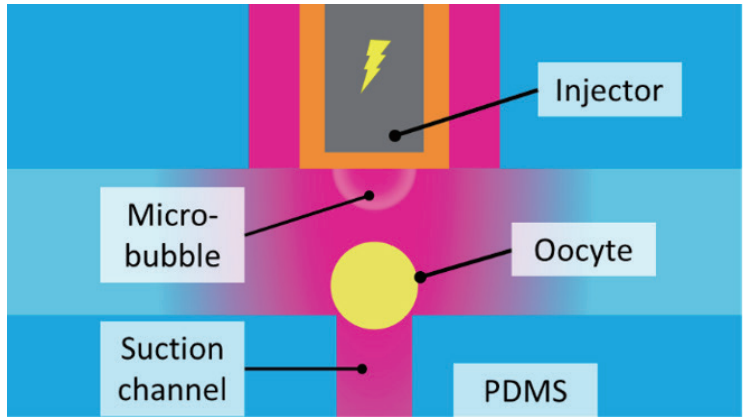

(a)



(b)

Fig. 1. (Color online) Model of bubble injection shown in two stages. (a) As a voltage difference is applied between the injector and the opposite electrode, small microbubbles are formed in front of the injector. (b) Then, the bubbles collapse near the oocyte, creating a sudden pressure change and a microjet. This microjet then makes a tiny hole on the cell membrane of the oocyte, and the injection solution is injected into the cell. 


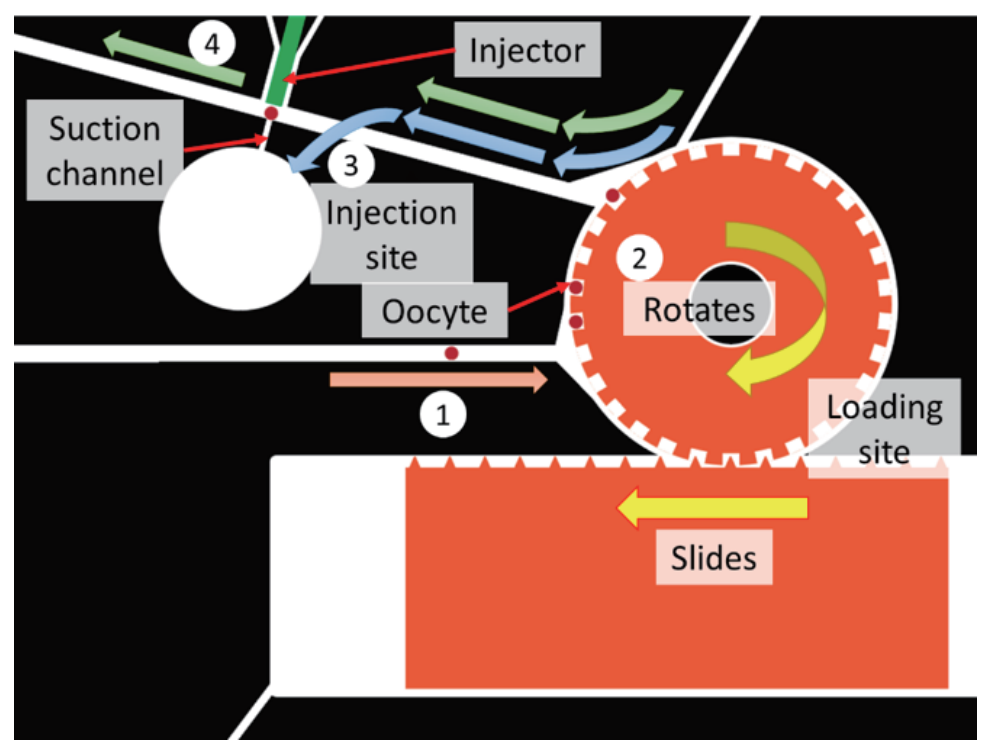

Fig. 2. (Color online) Model of entire loading and injection process, where the following steps are numbered in the figure. First, oocytes are inserted into the middle left channel and moved to the pinon following the pink arrow. Second, the oocytes enter the tooth gaps of the pinion and are transported to the upper microchannel individually by rotating the rack-pinion loader. Third, the oocytes are transported to the injection site and injected by the bubble injector. Fourth, the cells are released from the suction channel and removed from the chip. To realize these movements, the cells are moved along with the water flow, as shown by the pink, blue, and green arrows. In addition to transporting the cells, the control of the water flow and the slope near the pinion helps protect the cells by moving them slowly into the pinion and by removing them quickly from the pinion.

loader by the pressure of the syringe, as shown by the pink arrow in Fig. 2. Next, each of the cells enters a gap between the teeth of the pinion, separating them one by one. The rack then moves laterally, controlled by an external magnet, in order to rotate the pinion along with the cells to individually send them into the microchannel containing the injectors. Next, the cells are transported to the front of the injector by controlling the water flow (the words 'water', 'medium', and 'solution' are used interchangeably in this paper unless specified as 'pure water') inside the microchannel using a push-and-pull syringe pump configuration, as shown by the light blue and green arrows in Fig. 2. In order to transport the cells from the loader to the suction channel, the blue water flow is turned on, while the green water flow is turned off. Finally, as the cells are locked in place, they are injected by the bubble injector as also shown in Fig. 3 and then released to be stored by turning off the blue water flow and turning on the green water flow.

\section{Materials and Methods}

\subsection{Cell preparation}

The cells used in our experiments were bovine oocyte cells, sized between 100 and $150 \mu \mathrm{m}$. The cells were always kept inside a TCM199 medium to keep them fresh for a period of two weeks before they were renewed. Additionally, the TCM199 medium was always changed every day. 


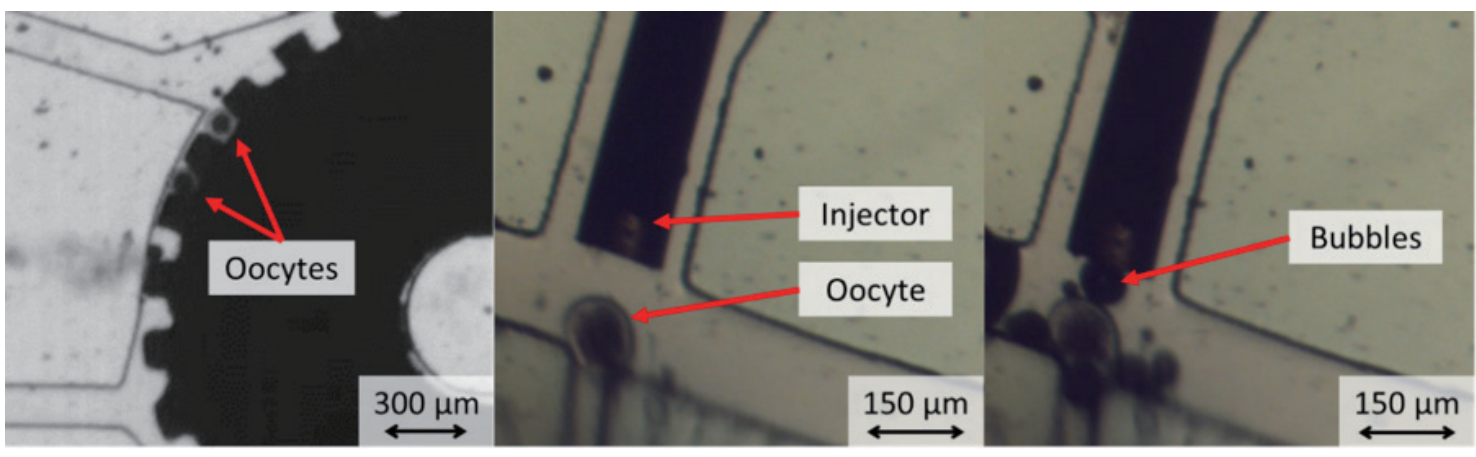

(a)

(b)

(c)

Fig. 3. (Color online) (a) Photograph of loading site, showing two oocytes being loaded by pinion. Additionally, as the loader can carry multiple cells in one cycle, the loader can move slowly without needing to move back and forth quickly while maintaining its throughput. (b) Photograph of oocyte before injection. (c) Photograph of oocyte after injection.

\subsection{Gel bead preparation}

For the loading experiment, soft gel beads were used instead of real cells, which were prepared as follows. First, calcium chloride powder from Kanto Chemical Co., Inc., and sodium alginate powder from FUJIFILM Wako Pure Chemicals Co. were prepared. Next, a calcium chloride solution was prepared by mixing $7.4 \mathrm{~g}$ of calcium chloride into $100 \mathrm{ml}$ of pure water. Then, sodium alginate solutions were prepared by mixing $0.75,1.0$, and $1.5 \mathrm{~g}$ of sodium alginate each into $100 \mathrm{ml}$ of pure water, which were used to make soft, medium, and hard gel beads, respectively. To be precise, the Young's moduli of the $1.0 \mathrm{~g} / 100 \mathrm{ml}$ and $1.5 \mathrm{~g} / 100 \mathrm{ml}$ gel beads were around 1.5 and 2.5 times that of the $0.75 \mathrm{~g} / 100 \mathrm{ml}$ gel beads, respectively. ${ }^{(23)}$ Next, each sodium alginate solution was sprayed into the calcium chloride solution separately, creating small spherical gel beads with various sizes and hardnesses. Finally, a $150 \mu \mathrm{m}$ filter was used to obtain gel beads with sizes smaller than $150 \mu \mathrm{m}$, which were then filtered with a $100 \mu \mathrm{m}$ filter to obtain gel beads sized between 100 and $150 \mu \mathrm{m}$, similar the bovine oocyte cell size.

\subsection{Component fabrication and assembly}

\subsubsection{Fabrication of PDMS chip}

To fabricate the chip, which has built-in microchannels and holds the other components, photolithography was performed using an SU-8 3050 epoxy-based photoresist in accordance with a previous study, as shown in Fig. $4 .{ }^{(24)}$ First, a $3 \times 3 \mathrm{~cm}^{2}$ silicon wafer was prepared by cleaning it with acetone, ethanol, and then pure water, each inside a $100 \mathrm{kHz}$ sonicator bath for 5 min. Then, the silicon wafer was spin-dried, which was followed by baking in an oven for $3 \mathrm{~h}$. Next, the photoresist was applied by spin-coating at $600 \mathrm{rpm}$ for $45 \mathrm{~s}$, then by increasing the rotation speed to $900 \mathrm{rpm}$ in $5 \mathrm{~s}$ and maintaining this speed for $45 \mathrm{~s}$. The photoresist-coated silicon wafer was then pre-heated at $95{ }^{\circ} \mathrm{C}$ for $1.5 \mathrm{~h}$. Then, the wafer was exposed to ultraviolet light with an energy of around $200 \mathrm{~mJ} / \mathrm{cm}^{2}$ from an unfiltered mercury lamp. The wafer was 


\section{Silicon wafer}

(1) Cleaning

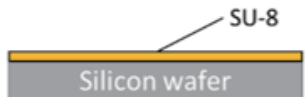

(2) SU-8 application by spin-coatìng

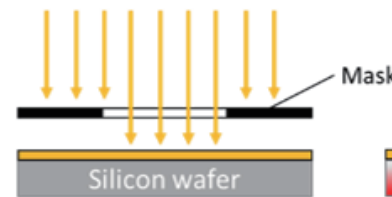

(5) Exposure through mask

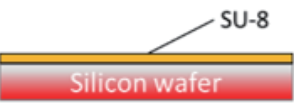

(6) Post-baking

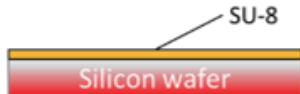

(3) Pre-heating

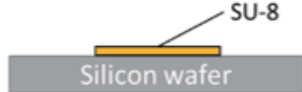

(7) Development

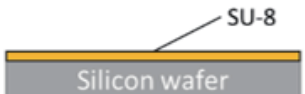

(4) Air cooling to room temperature

Fig. 4. (Color online) Steps of chip fabrication method. (1) The silicon wafer was cleaned with acetone, ethanol, and pure water. (2) SU-8 photoresist was applied to the silicon wafer by spin-coating at around $900 \mathrm{rpm}$ at room temperature and pressure. (3) The wafer was pre-heated for $1.5 \mathrm{~h}$. (4) The wafer was then air-cooled to room temperature. (5) The wafer was irradiated with ultraviolet light through the chip-shaped mask. (6) The chip was post-baked to bond the exposed SU-8 photoresist to the surface. (7) The wafer was developed inside a propylene glycol methyl ether acetate solution to obtain the fabricated chip.

then post-baked at $95{ }^{\circ} \mathrm{C}$ for $30 \mathrm{~min}$, developed using propylene glycol methyl ether acetate solution, and cleaned. After that, the height of the patterned photoresist was examined to ensure that it was between 130 and $150 \mu \mathrm{m}$ across the wafer.

To create the chip itself, the patterned silicon wafer was used to mold PDMS, as described in the following steps. First, a mixed PDMS solution was prepared with a ratio ranging between 1:9.5 and 1:13. Then, the PDMS solution was poured onto the silicon wafer in a container and then vacuumed to remove any bubbles. Next, it was baked for $20 \mathrm{~min}$ at $95^{\circ} \mathrm{C}$ to solidify the PDMS. Finally, the PDMS was removed from the silicon wafer and it was determined that the pattern had transferred from the silicon wafer to the PDMS.

\subsubsection{Fabrication of rack-pinion loader}

To fabricate the rack-pinion loader, a combination of photolithography and electroplating based on previous research was used. ${ }^{(25)}$ First, gold was sputtered on a cover glass to create a gold layer, which was performed with $50 \mathrm{~mA}$ for $100 \mathrm{~s}$. Then, the same photolithography step as in Sect. 2.3 was performed on the gold-sputtered glass, excluding the cleaned part. Next, the developed glass was electroplated with nickel to a thickness of $90 \mu \mathrm{m}$, which was performed at $3 \mathrm{~mA} / \mathrm{dm}^{2} \mu \mathrm{m}$. Finally, the fabricated rack-pinion loader was removed from the glass cover.

In this study, we used the following specifications for the rack-pinion loader. First, the rack-pinion tooth gap was $150 \mu \mathrm{m}$ and the height of each tooth was $120 \mu \mathrm{m}$. This is because the maximum size of bovine oocytes ranges from 100 to $150 \mu \mathrm{m}$, and with these dimensions, the largest oocyte cell can still be loaded without problem while still preventing two of the smallest oocytes from entering a single tooth gap. The width of a tooth was also set to $150 \mu \mathrm{m}$. This is because the limited resolution of electroplating made it difficult to fabricate pinion teeth narrower than $150 \mu \mathrm{m}$. However, narrower teeth are generally preferable to increase the maximum loading capacity in one cycle. The diameter of the pinion was set to $3 \mathrm{~mm}$, which is 
the largest possible diameter that does not interfere with other holes and microchannels. The hole diameter of the pinion was set to $750 \mu \mathrm{m}$, which is the smallest possible diameter for the PDMS shaft to bond with the glass. Generally, the diameter should be made as large as possible to maximize the transferred torque, and the hole should be made as small as possible to reduce the resistance from the friction. Finally, the height of each tooth of the rack was set to $120 \mu \mathrm{m}$ with a $150 \mu \mathrm{m}$ gap to match the profile of the pinion. The size of the rack was made as large as possible without interfering with the other components. The rack was made $2 \mathrm{~mm}$ high and $4.8 \mathrm{~mm}$ wide, allowing it to accommodate 16 teeth. With these specifications, one stroke or one cycle is capable of transporting nine cells at a time as 12 of the rack teeth can touch the pinion in one cycle and the minimum travel distance for each cell is equal to three teeth.

\subsubsection{Fabrication of bubble injector}

The bubble injector was fabricated using a tungsten wire and a polyimide tube, which were assembled by the following steps. First, a tungsten wire of $100 \mu \mathrm{m}$ diameter was inserted into a polyimide tube of $110 \mu \mathrm{m}$ inner diameter and $150 \mu \mathrm{m}$ outer diameter. Next, silver paste was applied to one end of the tungsten wire, covering the gap between the polyimide tube and the tungsten wire. Then, the tungsten wire was slid such that its end was between 0 and $30 \mu \mathrm{m}$ away from the polyimide tube. Finally, the end of the tungsten wire closer to the applied silver paste was heated for at least $30 \mathrm{~s}$ using a solder iron to seal the air gap between the wire and the tube.

\subsubsection{Chip assembly}

After fabricating all of the components, the chip was assembled with the layout shown in Fig. 5 by the following few steps. Initially, all four sides of the PDMS cast were cut in order

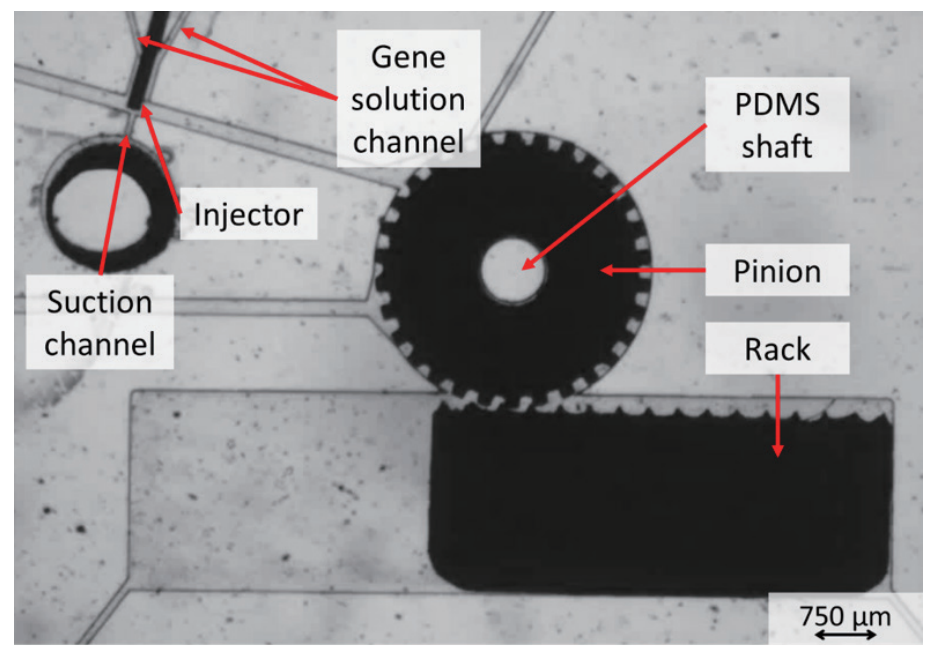

Fig. 5. (Color online) Assembly layout for injector, pinion, and rack. The injector is positioned in front of the suction channel between the gene solution channels. The hole of the pinion is inserted into the shaft made of PDMS and the rack is inserted into the case underneath the pinion. 
to ensure that the PDMS surface could later be bonded properly to the glass without any gap. Then, a $1.5 \mathrm{~mL}$ biopsy punch was used to make the holes on the PDMS cast, whose positions are shown in Fig. 6. Next, a $3 \times 3 \mathrm{~cm}^{2}$ glass cover and the PDMS cast were bombarded with plasma. Then, the rack-pinion loader and the bubble injector were placed inside the PDMS according to the layout shown in Fig. 6. Finally, the glass cover was placed onto the PDMS, sealing all of the components inside, and the fabricated chip was heated on a hot plate at $140{ }^{\circ} \mathrm{C}$ for 10 min to permanently bond the glass and PDMS, similarly to that in other studies. ${ }^{(26)}$

\subsection{Device setup and operational method}

\subsubsection{Microchannel flow and syringe pump setup}

In order to properly control the cell movements inside the chip, it is important to control the fluid movement inside the chip and, thus, the following setup was designed. First, the holes in the chip layout were categorized into the following seven different categories as shown in Fig. 6:

1. Water input to flush cells when they are stuck inside hole 2, where the pressure is applied by manually pushing the syringe.

2. Cell and water input, where the pressure is applied by manually pushing the syringe.

3. Air ejection hole, which was used to remove air from the chip to prevent the direct exposure of the cells to air.

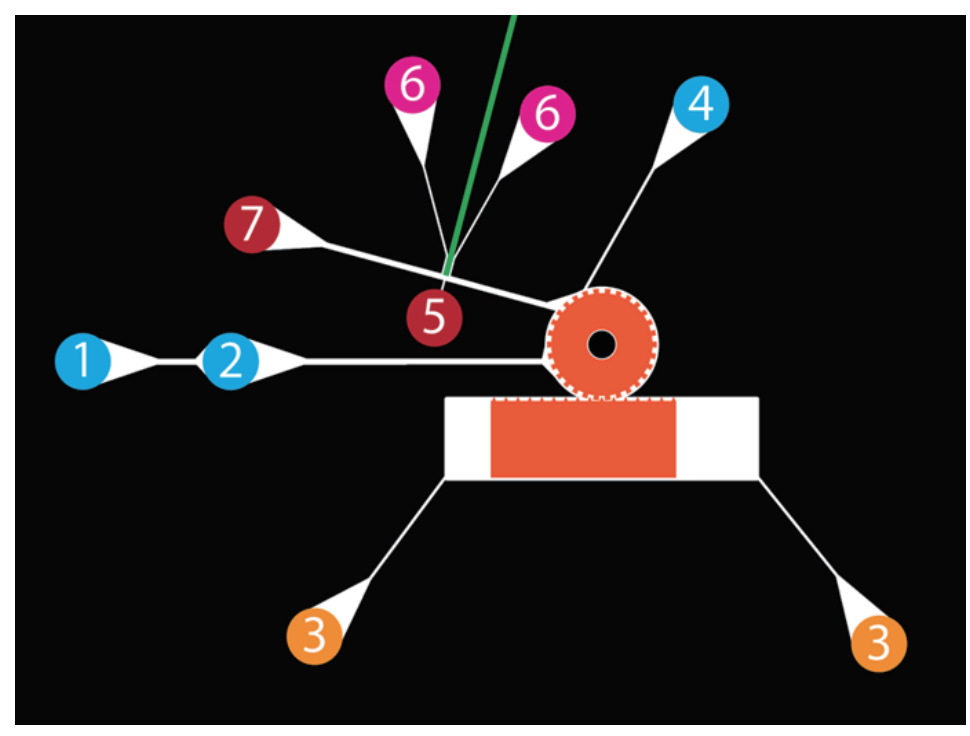

Fig. 6. (Color online) Water flow layout with color-coded circles, indicating the solution entering and leaving each circle. Nos. 1, 2, and 4 of the blue circles indicate insertion holes, where water or solution is inserted into the chip. No. 3 of the orange circles at the bottom indicates air ejection holes, where air is extracted during the preparation stage. No. 6 of the pink circles indicates the hole for gene solution insertion, and finally, nos. 5 and 7 of the red holes indicate the water or solution output, where the water or solution is extracted by a syringe pump outside the chip. The orange tool in the middle is the rack-pinion loader and the green line is the bubble injector. 
4. Water input to create a water flow from holes 4 to 5 and from holes 4 to 7 , with flow rates of $5 \mathrm{~mL} / \mathrm{h}$ for moving the cell to the front of the injector and $20 \mathrm{~mL} / \mathrm{h}$ for removing the cell from the chip.

5. Water output for the suction mechanism, designed to lock down the cells to be injected in front of the injector. Additionally, the opposite electrode of the bubble injector is also inserted into this hole.

6. Gene solution input, where the pressure is applied by manually pushing the syringe.

7. Water output to remove the cells from the chip after injection, with the same flow rate as hole 4.

Additionally, holes 4, 5, and 7 were connected by two push-pull syringe pumps outside of the chip, which push and pull the syringes at the same rate to create a constant flow rate. The first pump connects holes 4 and 5 as well as holes 4 and 7, with a permanent push configuration into hole 4 and a valve to switch between holes 5 and 7. This allows the first pump to either move the cell from the pinion to the suction channel or move the cell from the suction channel or the pinion directly from outside the chip. The second pump connects holes 4 and 7 directly, allowing water to flow while the first pump is still locking the cell in front of the injector. This allows bubbles or dirt to be removed from the channel while still locking the cell in front of the injector.

\subsubsection{Rack-pinion loading method}

To load the cells using the rack-pinion loader, the rack needs to be moved sideways, which can be controlled from outside the chip using an external magnet as shown in Fig. 7. In this case, as the magnet is moved sideways, the rack is also pulled sideways, which in turn rotates the pinion. Other works have shown that if the magnetic field is parallel to the magnetic tool, there is an equilibrium point where the friction and magnetic forces are equal. ${ }^{(27)}$ That is, the position of the rack directly corresponds to that of the external magnet, allowing a simple

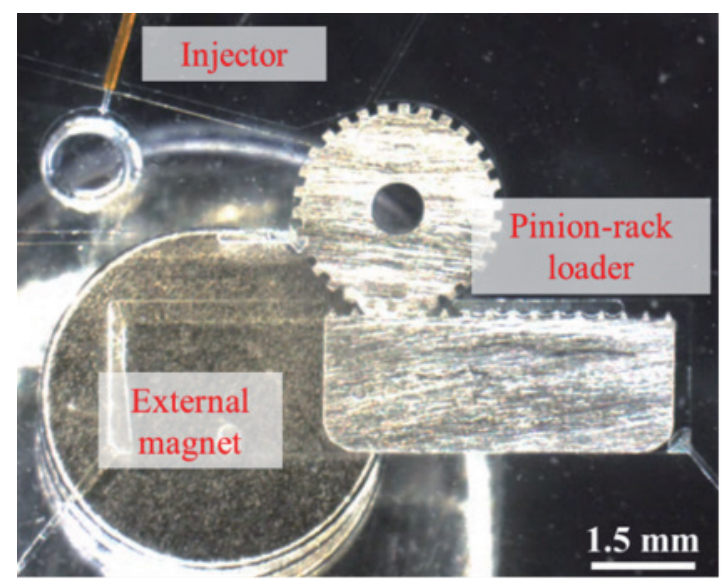

Fig. 7. (Color online) Photograph showing the external magnet used to control the rack. The magnet used has a strength of $400 \mathrm{mT}$ and a diameter of $5 \mathrm{~mm}$, and is driven manually by the operator. 
and accurate control of the rack-pinion loader. Additionally, since the pinion and the rack are designed not to slip, the loading can be performed in a single stroke.

\subsubsection{Electrical circuit setup of bubble injector}

To ensure that the bubble injector works properly, the electrical circuit of the bubble injector, which is explained next, was set up between the injector and the opposite electrode. First, a high-frequency and high-voltage pulsed DC generator power supply was prepared. Next, the negative end of the power supply was connected to the bubble injector and the positive end to the opposite electrode. Then, an electrolyte solution was inserted into the system so that it was always connected the bubble injector and the electrode. Finally, cycles with voltages ranging from 1000 to $1900 \mathrm{~V}$ were applied between the two electrodes for 5 to $20 \mu \mathrm{s} 5$ to 10 times at $1 \mathrm{~ms}$ intervals.

\subsection{Experimental setup for loader tests}

\subsubsection{Strength test method}

A strength test was performed to test the maximum force output of the loader, which involved the following steps, as shown in Fig. 8. First, the stage shown in Fig. 8(a) was prepared, and the chip, plastic cantilever beam, tungsten needle, and load cell were set up on the stage as shown in Fig. 8(b). Next, the strength test was performed by moving the magnet down to transfer the force to the needle, cantilever beam, and load cell. Then, the maximum

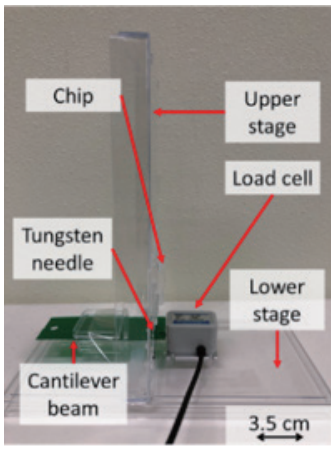

(a)

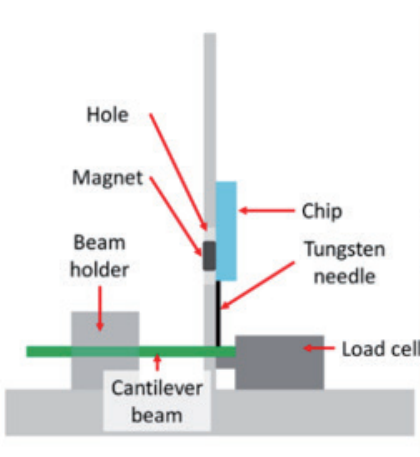

(b)

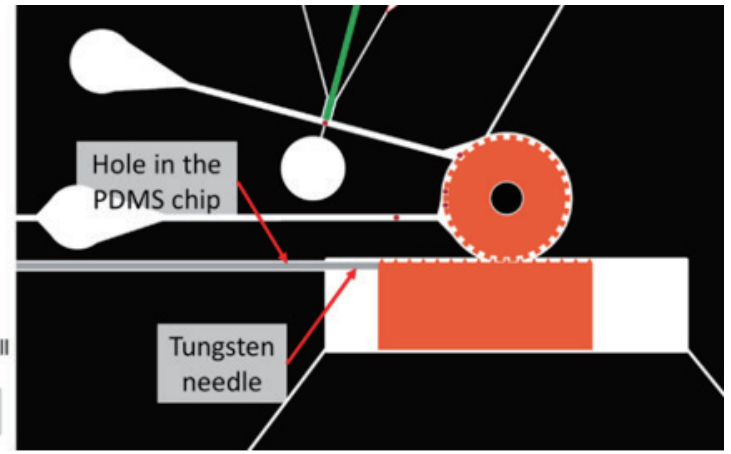

(c)

Fig. 8. (Color online) Photograph and model of strength test setup. (a) Photograph of actual stage used to measure force output of loader. The apparatus used includes the chip itself, a stage, a tungsten needle, a load cell, a cantilever beam, and a cantilever beam holder. The stage is composed of two stiff plastic covers that had been melted and bonded together to form the illustrated $\mathrm{T}$ shape. (b) Cross-sectional view of model of strength test setup. The hole on the upper stage allows a magnet to touch the back of the chip directly, enabling the transmission of a realistic force from the magnet to the loader. This force pushes the tungsten needle underneath it along with the cantilever beam and load cell, and the force is output as a voltage. (c) Model of tungsten needle installed inside the chip. A hole is made on the side of the PDMS chip to allow the tungsten needle to directly touch the rack for direct force transfer. 
voltage output was measured using the load cell when it stabilized. Following this, the chip was removed from the stage and replaced with known weights until the load cell reading matched the maximum voltage output generated by the chip. In this way, the maximum force could be represented by calculating the weight of the equivalent mass.

\subsubsection{Loading test method}

The loading test was designed to evaluate the number of cells delivered and the time required to finish one cycle, and was based on the following steps. First, the soft, medium, and hard gel beads described in Sect. 2.2 were prepared. Then, the gel beads were inserted into the chip to be loaded. Next, a single full cycle was performed to load the beads, and the time taken to deliver them and move back to the initial position was measured. This was carried out four times for each bead hardness at flow rates of 5, 12.5, and $20 \mathrm{~mL} / \mathrm{h}$ for flow 3 in Fig. 2.

\subsection{Experimental setup for cell injection test}

Although the injection capability of the bubble injector has been confirmed outside the chip,${ }^{(9)}$ its capability under the limitations of the PDMS chip has yet to be confirmed. Therefore, in this test, the injection capability of the bubble injector inside the chip was confirmed using the following steps. First, the cells were inserted into the chip to be loaded and positioned in front of the bubble injector, which was performed by extracting water from the suction channel in front of the injector. Then, a medium with blue fluorescent beads of $2.1 \mu \mathrm{m}$ size was inserted between the injector and the cells. Next, the cells were injected 10 times with a voltage pulse of $1500 \mathrm{~V}$ for $10 \mu \mathrm{s}$ at $1 \mu \mathrm{s}$ time intervals between pulses. These settings were used since they were the lowest possible input voltage and duration for which successful injection in the chip was confirmed. Finally, the injected cells were extracted, washed, and observed under a microscope with the help of ultraviolet light to see whether the beads were injected into the cells.

\section{Results and Discussion}

\subsection{Loader capability}

According to the results of strength, precision, and loading tests, the loader outputs a large force and has high precision and throughput capability. First, the rack-pinion loader outputs a large force of $40 \mathrm{mN}$, which was reduced by the wall friction inside the system. That is, the rack-pinion loader can operate even under harsh conditions, such as in the presence of bubbles or a dirt-filled chip as shown in Figs. 9(a)-9(c). This allows bubbles and dirt to be moved to the upper channel and removed from the system by water flow 4 shown in Fig. 2, enabling the operation conditions to return to normal. Additionally, the rack-pinion loader also has good control with 0.8 pinion tooth accuracy, which is equivalent to an accuracy of around $3^{\circ}$. This means that the rack-pinion loader is sufficiently reliable to load cells individually to the 


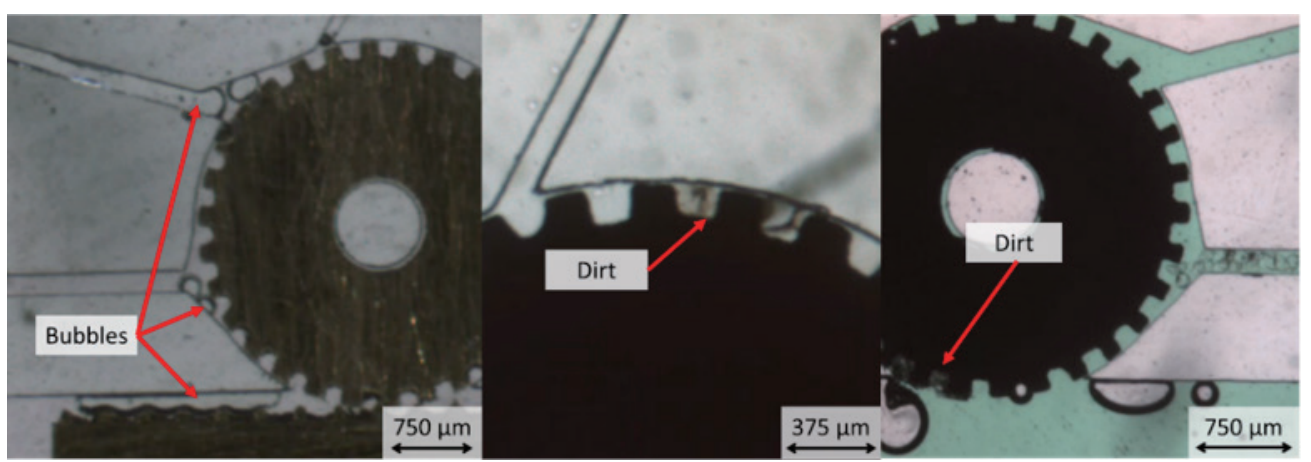

(a)

(b)

(c)

Fig. 9. (Color online) Photographs showing reliable operation of rack-pinion loader under harsh conditions. Although the chip should ideally be filled with a bubble-free clean medium, in reality, bubbles and dirt exist, making it important for a reliable loader to be able to operate under the following harsh conditions: (a) with bubbles inside the chip; (b) (c) rack-pinion loader with dirt stuck on the pinion tooth.

injector every time without a negative feedback system, thus enabling a system for automatic cell loading to be developed more easily in the future. Moreover, the rack-pinion loader is also capable of finishing a cycle in 6,8 , and $12 \mathrm{~s}$ on average for hard, medium, and soft gel beads, respectively. This allows the loader to load up to an average of 42 gel beads per min, which means that bottlenecks will not occur in the current system. Detailed data are shown in Tables 1-3.

From Tables 1-3, several conclusions can be drawn regarding the loading efficiency. First, harder particles can be loaded significantly faster than softer particles. This might have been because softer particles tend to have a higher friction coefficient with the sides of the pinion teeth, making it harder to remove softer particles. Second, a higher flow rate generally increases the loading efficiency. Similarly, this might have also been caused by the increased flow rate applying a larger force to overcome the friction on the gel beads. The soft gel beads, however, do not seem to be affected by the increased flow rate. This might have been for several reasons, such as the beads being stuck in the pinion and fewer beads being delivered per cycle. Similar to the problem of bubbles and dirt, stuck beads can be released by flushing the top channel with a strong water flow (flow 4), which will restore the maximum loading capacity in the next cycle.

Nevertheless, the rack-pinion loader has yet to achieve its maximum potential and still has room for improvement. First, the current fitting of the pinion is still too loose, allowing it to sometimes touch the walls, increasing the overall friction. Future developments should focus on finding the ideal clearance between the walls, shaft, pinion, and rack. Second, the rack-pinion loader can also be used for operations other than loading, such as sorting. This means that using the unique potential of the rack-pinion loader as a powerful sorting tool will allow the chip to sort larger and heavier objects, such as to remove dirt and bubbles from the system. Third, as the loader itself is made of metal, it has the potential to act as an electrode for electroporation-type intracellular delivery. This is important because although microinjection 
Table 1

Loading efficiency data from loading test on gel beads with different hardnesses with $20.0 \mathrm{~mL} / \mathrm{h}$ flow rate along blue and green arrows in Fig. 2.

\begin{tabular}{lccc}
\hline $20.0 \mathrm{~mL} / \mathrm{h}$ & Soft gel beads $\left(\mathrm{bpm}^{*}\right)$ & Medium gel beads $\left(\mathrm{bpm}^{*}\right)$ & Hard gel beads $\left(\mathrm{bpm}{ }^{*}\right)$ \\
\hline Minimum & 7.5 & 15.0 & 24.0 \\
Maximum & 12.0 & 45.0 & 60.0 \\
Average & 10.9 & 27.4 & 42.0
\end{tabular}

${ }^{*}$ bpm $=$ beads per minute, calculated by dividing the number of beads loaded per cycle by the time taken to finish the cycle. Values have been rounded to one decimal place.

Table 2

Loading efficiency data from loading test on gel beads with different hardnesses with $12.5 \mathrm{~mL} / \mathrm{h}$ flow rate along blue and green arrows in Fig. 2.

\begin{tabular}{lccc}
\hline $12.5 \mathrm{~mL} / \mathrm{h}$ & Soft gel beads $(\mathrm{bpm})$ & Medium gel beads $(\mathrm{bpm})$ & Hard gel beads $(\mathrm{bpm})$ \\
\hline Minimum & 4.0 & 13.3 & 24.0 \\
Maximum & 10.0 & 30.0 & 48.0 \\
Average & 6.3 & 18.3 & 40.0 \\
\hline
\end{tabular}

Table 3

Loading efficiency data from loading test on gel beads with various hardnesses with $5.0 \mathrm{~mL} / \mathrm{h}$ flow rate along blue and green arrows in Fig. 2.

\begin{tabular}{lccc}
\hline $5.0 \mathrm{~mL} / \mathrm{h}$ & Soft gel beads (bpm) & Medium gel beads (bpm) & Hard gel beads (bpm) \\
\hline Minimum & 6.9 & 10.0 & 17.1 \\
Maximum & 12.0 & 15.0 & 36.0 \\
Average & 9.2 & 12.2 & 25.7 \\
\hline
\end{tabular}

tools, such as bubble injectors, are straightforward and simple, electroporation is ideal for small cargo delivery, and combining it with the rack-pinion tool would allow close-proximity singlecell electroporation.

\subsection{Injector capability}

Through our experiment, it was confirmed that the injector is applicable for the chip with a function to introduce a substance into a target, and fluorescent beads were observed in the injected cells, as shown in Fig. 10. First, a hole can be seen on the cell membrane in Fig. 10(a), which is most likely caused by the bubble injector since the other components cannot make such a small hole. Next, a small glowing fluorescent bead can be seen inside the same cell in Fig. 10(b). This bead is likely to be inside the cell as its image is focused on the same plane as the cell.

After the observation of 40 cell injections in the chip, the injection efficiency was discovered to be 80 to $87.5 \%$ by the analysis of individual injected cells as shown in Fig. 10. The upper success rate of $87.5 \%$ was obtained by averaging the successful injections including the situation shown in Fig. 10(c). Meanwhile, the lower success rate of $80 \%$ was obtained by averaging the successful injections excluding the situations shown in Fig. 10(c). Therefore, it was confirmed that the injection success rate is between 80 and $87.5 \%$. 


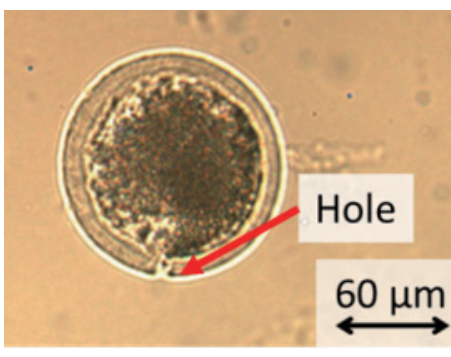

(a)

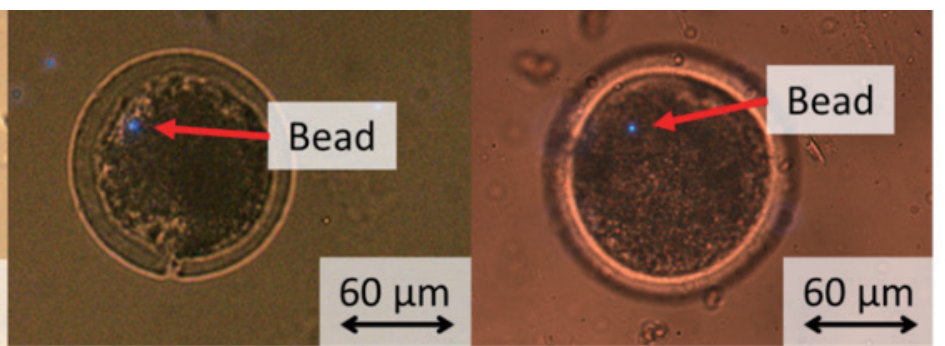

(b)

(c)

Fig. 10. (Color online) Pictures showing injected cell. (a) Photograph showing hole created by bubble injector. (b) Photograph showing fluorescent bead inside cell under ultraviolet light. (c) Situation where it is unclear whether a bead is inside or not; although the bead is inside the cell membrane, the membrane is out of focus.

Likewise, the injector can still be improved by quantifying the injection power through two main areas of research. The first is to discover the relationship among the applied voltage, its duration, and the success rate of the injection. This is important as increasing both the magnitude of the applied voltage and its duration causes more energy to be transferred, which in turn increases the injection power. The second is to discover the relationship among the number of voltage pulses applied, the time gap between the pulses, and the success rate of the injection. By clarifying these relationships, the injection power can be better controlled, thus increasing the success rate of the injections.

\subsection{Overall chip performance}

In our experiment, it was confirmed that the fabricated chip is simple and efficient, which leads to an easier operation without any intensive training requirements. Because the chip combines a one-DOF rack-pinion loader with a bubble injector, the control of each component is easy and straightforward. That is, the entire process of loading, holding the cell in front of the injector, and injection was performed in just under $10 \mathrm{~s}$, or at a speed of 6 cells per min, rivaling the high throughput of 7 cells processed per min of commercial electroporation and that of 4 cells per min of other in-chip microinjection chips. ${ }^{(28,29)}$ In the future, this chip can also be manipulated automatically owing to its simple controls for loading and injection, which will further reduce the loading and injection times. To be precise, the loading time can be reduced down to $2 \mathrm{~s}$ and the injection time can be reduced down to $0.1 \mathrm{~s}$ with a combined processing time of $2.1 \mathrm{~s}$, which is comparable to other similar microinjection chips with an expected $1 \mathrm{~s}$ injection time. ${ }^{(16)}$ Additionally, the system can also load multiple cells in one cycle, allowing a continuous supply of cells for the injector, as shown in Figs. 11 and 12. However, during the experiment, both the injection setting input and the microtool actuation were performed manually, resulting in a significantly longer time. Nevertheless, this chip can be used by a nonexpert with little prior training, as it only requires the operator to slide a magnet and push a few buttons, while still maintaining a competitive throughput.

In the future, there are two main improvements that could be made to further simplify and increase the efficiency of the system. First, the width of the suction channel should be reduced 


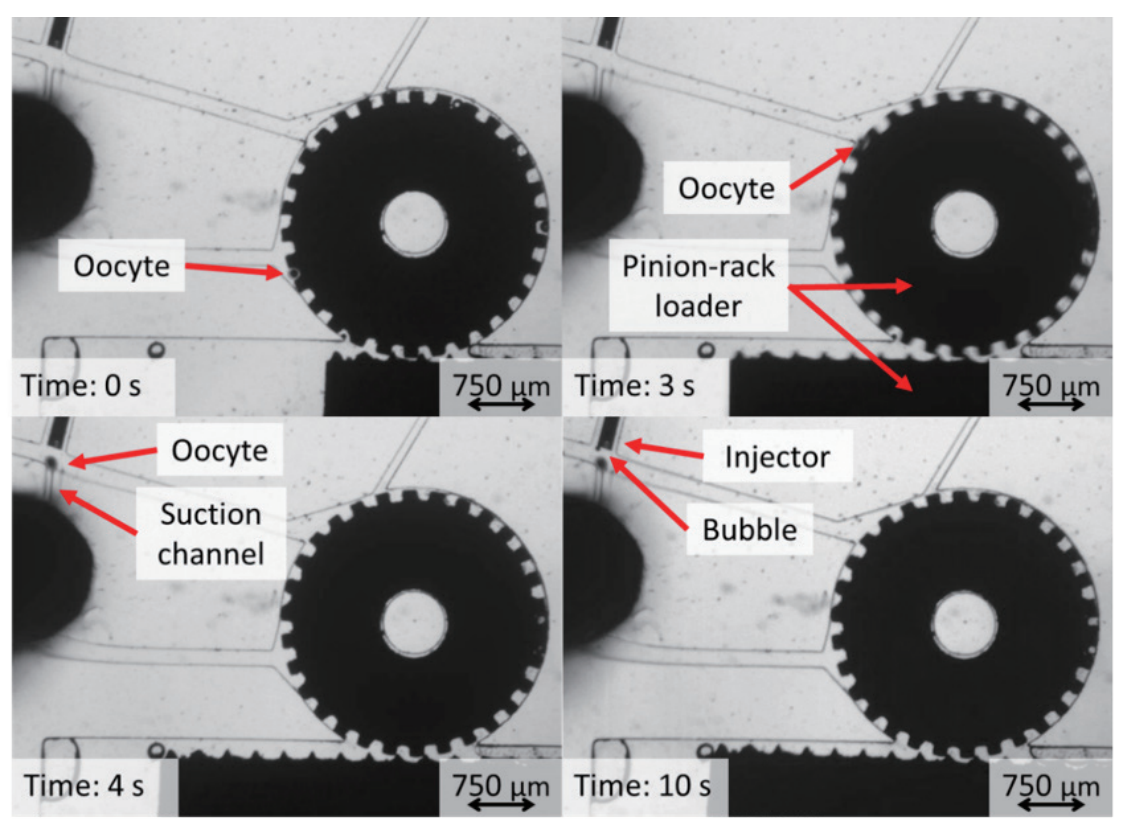

Fig. 11. (Color online) Series of photographs following loading and injection of oocyte. At $0 \mathrm{~s}$, the oocyte has just entered a tooth gap of the pinion. At $3 \mathrm{~s}$, the oocyte has been loaded to the upper microchannel. At $4 \mathrm{~s}$, the oocyte in front of the injector is extracted by the suction channel in less than $1 \mathrm{~s}$. At $10 \mathrm{~s}$, the oocyte is injected by the bubble injector, which takes less than $6 \mathrm{~s}$. Here, a few remaining bubbles can be seen around the oocyte.

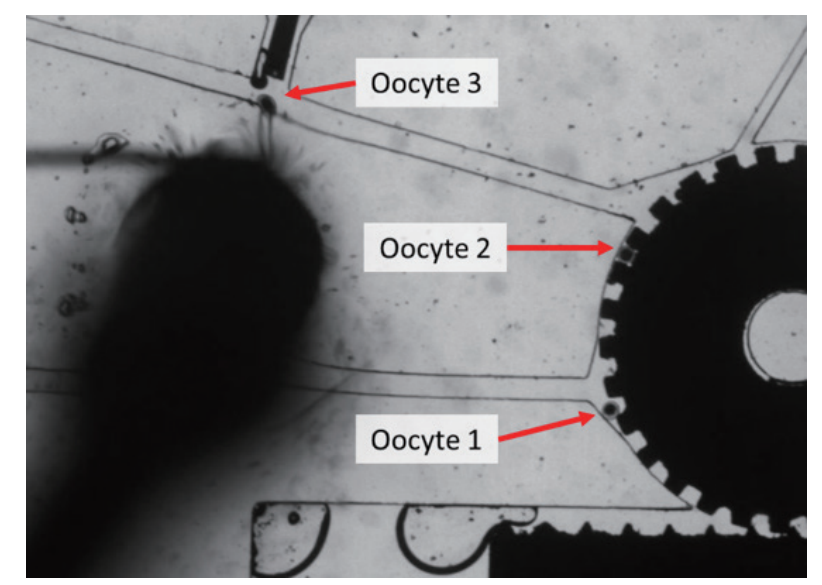

Fig. 12. (Color online) Proof-of-concept photograph showing the intended application of the system. While oocyte 3 is being injected, oocyte 2 is already ready to be sent to the injection site and oocyte 1 is ready to be loaded. This concept will significantly improve the processing time of cell injections.

to $15 \mu \mathrm{m}$ or smaller. In this experiment, it was apparent that the suction channel in front of the injector, which is currently $50 \mu \mathrm{m}$ wide, is still too large for the oocytes. This can sometimes cause the cells to be pulled into the suction channel. Second, a continuous flow is required for both the cell insertion flow and the gene solution flow. The current chip design does not take into account the outflow for gene solution insertion and cell insertion, which generates 
a positive pressure inside the chip and causes the water flow in the chip to deviate from the expected behavior. This can be solved by adding more holes to remove the water and to better control the water flow inside the chip.

\section{Conclusions}

In this paper, we have introduced a novel chip, which combines a rack-pinion loader and a bubble injector, that will simplify cell injection and enable it to be performed by non-experts. The rack-pinion loader showed a force of $40 \mathrm{mN}$, an accuracy of $3^{\circ}$, and an average loading speed of up to 42 beads per minute. In other words, the loader was powerful enough to work under harsh conditions while maintaining a satisfactory precision and medium-loading speed. The bubble injector was confirmed to work properly inside the chip as fluorescent beads were observed in injected bovine oocytes with a success rate of 80 to $87.5 \%$. Additionally, the combination of these components has allowed a simple operation from loading to injection in under $10 \mathrm{~s}$, which allows the chip to process at least 6 cells per min. As a result, it is possible for untrained people to carry out cell injections with this chip, along with automated cell injection, both of which will improve the accessibility of cell injection and support the rapid growth of biological fields.

\section{Acknowledgments}

This work was partly financed by the Ministry of Education, Culture, Sports, Science and Technology (grant number 19H02113). It was also supported by JST CREST (grant No. JPMJCR19S6), JST A-STEP (grant number AS3015134U), and AMED SENTAN (grant number JP17hm0102049). The authors are grateful to BEX Inc. and Setsuro Tech. Inc. for their valuable advice.

\section{References}

1 W. Xu: Microinjection: Methods and Protocols Methods in Molecular Biology (Springer, 2019) p. 1. https://doi. org/10.1007/978-1-4939-8831-0_1

2 Y. Yamanishi, S. Sakuma, T. Iyanagi, F. Arai, T. Arai, A. Hasegawa, T. Tanikawa, A. Ichikawa, O. Satoh, A. Nakayama, H. Aso, M. Goto, S. Takahashi, and K. Matsukawa: J. Robot. Mechatron. 22 (2010) 371. https://doi. org/10.20965/jrm.2010.p0371

3 M. Hashimoto and T. Takemoto: Sci. Rep. 5 (2015) 11315. https://doi.org/10.1038/srep11315

4 K. Kim and W. G. Lee: J. Mater. Chem. B 5 (2017) 2726. https://doi.org/10.1039/C7TB00038C

5 W. Wang, P. M. Kutny, S. L. Byers, C. J. Longstaff, M. J. DaCosta, C. Pang, Y. Zhang, R. A. Taft, F. W. Buaas, and H. Wang: J. Genet. Genomics 43 (2016) 319. https://doi.org/10.1016/j.jgg.2016.02.004

6 F. Iwata, K. Yamazaki, K. Ishizaki, and T. Ushiki: Jpn. J. Appl. Phys. 53 (2014) 36701. https://doi.org/10.7567/ JJAP.53.036701

7 S. Kasparov, A. G. Teschemacher, D.-Y. Hwang, K.-S. Kim, T. Lonergan, and J. F. R. Paton: Prog. Biophys. Mol. Biol. 84 (2004) 251. https://doi.org/10.1016/j.pbiomolbio.2003.11.011

8 T.-H. Wu, T. Teslaa, S. Kalim, C. T. French, S. Moghadam, R. Wall, J. F. Miller, O. N. Witte, M. A. Teitell, and P. Y. Chiou: Anal. Chem. 83 (2011) 1321. https://doi.org/10.1021/ac102532w

9 K. Takahashi, S. Omi, and Y. Yamanishi: 2015 28th IEEE Int. Conf. Micro Electro Mechanical Systems (MEMS) (2015) 655. https://doi.org/10.1109/MEMSYS.2015.7051042 
10 S. I. Sukharev, A. V. Titomirov, and V. A. Klenchin: Gene Therapeutics: Methods and Applications of Direct Gene Transfer (Birkhäuser, 1994) p. 210 https://doi.org/10.1007/978-1-4684-6822-9_12

11 J. Shi, Y. Ma, J. Zhu, Y. Chen, Y. Sun, Y. Yao, Z. Yang, and J. Xie: Molecules 23 (2018) 3044. https://doi. org/10.3390/molecules23113044

12 H. Kuriki, Y. Yamanishi, S. Sakuma, S. Akagi, and F. Arai: 2013 IEEE 26th Int. Conf. Micro Electro Mechanical Systems (MEMS) (IEEE, 2013) 209. https://doi.org/10.1109/MEMSYS.2013.6474214

13 S. Itabashi, T. Kambayashi, T. Shimamura, K. Takahashi, and Y. Yamanishi: 2016 IEEE 11th Annu. Int. Conf. Nano/Micro Engineered and Molecular Systems (NEMS) (2016) 604. https://doi.org/10.1109/ NEMS.2016.7758324

14 Y. Zhao, H. Sun, X. Sha, L. Gu, Z. Zhan, and W. J. Li: Micromachines 10 (2019) 7. https://doi.org/10.3390/ mi10010007

15 W. G. Lee, U. Demirci, and A. Khademhosseini: Integr. Biol. (Camb). 1 (2009) 242. https://doi.org/10.1039/ b819201d

16 A. Adamo and K. F. Jensen: Lab Chip 8 (2008) 1258. https://doi.org/10.1039/B803212B

17 S. Sakuma, Y. Kasai, T. Hayakawa, and F. Arai: Lab Chip 17 (2017) 2760. https://doi.org/10.1039/C7LC00536A

18 Y. Yamanishi, S. Sakuma, and F. Arai: J. Robot. Mechatronics 20 (2008) 273. https://doi.org/10.20965/ jrm.2008.p0273

19 A. Waldschik, M. Feldmann, V. Seidemann, and S. Büttgenbach: Design and Manufacturing of Active Microsystems Microtechnology and MEMS (Springer, 2011) p. 207. https://doi.org/10.1007/978-3-642-12903$2 \_12$

20 Y. Yamanishi, S. Sakuma, Y. Kihara, and F. Arai: J. Microelectromech. Syst. 19 (2010) 350. https://doi. org/10.1109/JMEMS.2010.2041188

21 K. Ichikawa, S. Maeda, and Y. Yamanishi: J Microelectromech Syst. 27 (2018) 305. https://ieeexplore.ieee.org/ document $/ 8287817$

22 H. Kuriki, Y. Yamanishi, S. Sakuma, S. Akagi, and F. Arai: J. Robot. Mechatron. 25 (2013) 476. https://doi. org/10.20965/jrm.2013.p0476

23 M. Mancini, M. Moresi, and R. Rancini: J. Food Eng. 39 (1999) 369. https://doi.org/10.1016/S02608774(99)00022-9

24 L. Feng, T. Kawahara, Y. Yamanishi, M. Hagiwara, K. Kosuge, and F. Arai: J. Robot. Mechatron. 24 (2012) 133. https://doi.org/10.20965/jrm.2012.p0133

25 N. Inomata, T. Mizunuma, Y. Yamanishi, and F. Arai: J. Microelectromech. Syst. 20 (2011) 383. https://doi. org/10.1109/JMEMS.2010.2100028

26 Y. Yamanishi, T. Kawahara, T. Iyanagi, M. Hagiwara, T. Mizunuma, N. Inomata, S. Kudo, and F. Arai: J. Robot. Mechatron. 22 (2010) 623. https://doi.org/10.20965/jrm.2010.p0623

27 M. Hagiwara, T. Kawahara, Y. Yamanishi, and F. Arai: Adv. Robot. 25 (2011) 991. https://doi. org/10.1163/016918611X568611

28 L. Setsurotech Co.: https://www.setsurotech.com/genome-editing/mouse/ (accessed September 2019).

29 A. Noori, P. R. Selvaganapathy, and J. Wilson: Lab Chip 9 (2009) 3202. https://doi.org/10.1039/b909961a 